\title{
DELICATE STATE OF MENTAL HEALTH AND ITS IMPACT ON HEALTHCARE WORKERS DURING THE COVID-19 PANDEMIC: A REVIEW
}

\author{
Dr. Pooja Malhotra* *Corresponding Author \\ Jashandeep Singh
}

ABSTRACT Introduction: The novel corona virus (COVID-19), a pandemic, spread to around 198 countries, and has affected millions of people globally. The associated morbidity and mortality challenged the nations in several ways. One such unaddressed area is the mental health impact on the healthcare workers and staff during this pandemic. The review aims at summarizing the evidence of mental health issues of COVID-19 pandemic on the frontline healthcare workers (HCW's).

Materials And Methods: A review of the literature of the mental health issues faced by the healthcare workers during the pandemic was done. Literature search was conducted in the following databases: PubMed, Google Scholar, Embase; articles relevant to the subject, in the duration of last 8 months (Jan-2020 to Aug-2020) were reviewed. We have highlighted the most relevant data concerning the disease characteristics, personal and organizational factors that may have contributed to development of psychological changes, distress and mental health symptoms.

Results: There is a generalized climate of wariness and uncertainty, particularly amongst the health professionals, which has been provoked by the disease characteristics of the current COVID-19 pandemic and various sociodemographic variables. Other factors included, rapid spread of disease, severity of symptoms, lack of knowledge of the disease, organizational factors and death among healthcare professionals. The mental health problems varied with respect to the sociodemographic variables like, gender, profession, age, place of work, social support and department of work. Evidence suggests that COVID19 may be an independent risk factor for increase in stress, anxiety, burnout and depression amongst the healthcare professionals, which could have long-term psychological implications.

Conclusion: It is of utmost importance to protect and ensure the mental health of the healthcare professionals, to address large scale health crisis. Therefore, planning of future prevention strategies is essential to promote mental well-being. Implementation to bolster response and prevention strategies by training healthcare professionals on crisis management and mental help. Evaluation of mental health status should be done by regular screening of the personnel by the multidisciplinary Psychiatry teams.

KEYWORDS : COVID-19, Healthcare workers, Stress, Mental health, Anxiety, Depression, Occupational health, Health impact, Risk factors.

\section{INTRODUCTION:}

Novel coronavirus disease (COVID-19), since its inception in December-2019, has been spreading globally at a rapid rate. As in Dec-2019, COVID-19was first reported in the Hubei province of China, as a new severe type of pneumonia (Li et al., 2020; Zhu et al., 2020). Over the subsequent months, it rapidly extended around the world, however not all countries were equally affected. By 24-May-2020, the World Health Organization (WHO) reported 5,165,481 cases of COVID-19 including 336,430 deaths (WHO,2020). Amidst the crisis and exponential progression of this infectious disease throughout the world, our healthcare workers remain the main persons involved in the testing, screening and treatment of this condition.

Despite remaining crisis management personnel, the healthcare professionals are not themselves immune to the psychological consequences of the pandemic (Cai et al., 2020; Tam et al., 2004). Various previous researches on other infectious diseases, including those on Severe Acute Respiratory Syndrome (SARS), the Middle East Respiratory Syndrome (MERS) and the Ebola virus disease, consistently proved that the healthcare professionals reported symptoms of depression and anxiety, both during and after the outbreak, causing a severe and bold impact on their coping abilities and had long lasting effects in most cases (Duran et al., Bettinsoli et al.,). The frontline workers, those directly involved in handling the patients are at a greater risk than the others. The reason for such psychological impact includes excessive workload, increased working hours, inadequate social support, over-enthusiastic media news, inadequate personnel protective equipment and the most important of all, increased infection rate among the medical staff (Cai et al., 2020; Lee at al., 2020; Styra et al., 2020).

COVID-19, a pandemic, is a kind of a global war of humans against a disguised deadly virus and our frontline soldiers fighting on the front are our doctors and the paramedic staff. The whole medical fraternity frequently endures a multitude of mental challenges under this high pressure to survive and cure during this pandemic. (Li et al., 2020). The uncertainty over controlling the disease spread and magnitude of the multiple threats are amongst the most challenging circumstances during the crisis. On the other side, strains coupled with difficulties have worsened severe mental illness which includes anxiety, depression and thanatophobia amongst the healthcare workers (Zandifar and Badrfam, 2020).

The prompt reversal of role from HCW to a patient might lead to state of helplessness, stigma, adjustment issues, frustration and fear of discrimination in the medical staff (Rana et al., 2020). Literature that dates back to a decade ago, during the outbreak of SARS suggested that the HCW are at a higher risk of developing mental illness during these periods (Wu et al., $2005 a, b)$. A similar school of thought exists about the present COVID-19 pandemic, but there is a lack of systemic appraisal and critique on the existing studies.

\section{MATERIALS AND METHODS}

\subsection{Aim And Objectives}

The aim of this review is to summarize and to provide an overview of the current evidence- based information and research findings about the impact of the COVID-19 pandemic on the mental health of the health professionals. The objectives of the current review were to identify the relationship between the psychological, socio-demographic and disease (COVID-19) related variables and mental issues faced by the healthcare workers.

\subsection{Literature Searches}

The literature search was conducted in the following 
databases: PubMed, Medical Literature Analysis, Google Scholar, Embase and Retrieval System Online (MEDLINE), for all the relevant articles from January-2020 to August-2020. Search terms used for retrieving the articles were: psychological, stress, mental health, psychiatric issues, COVID-19, novel corona, corona, HCW, HP, medical staff, doctor, anxiety, depression, medical staff. Various articles, reviews, commentary, correspondence, letter to the editor and original research article relevant to the subject of the review were searched. Applicable reports were extracted using crossreferences.

\section{RESULTS}

During this period of crisis caused by the spread of Novel Corona Virus, all the medical professionals, especially those at the frontline of treating the affected patients, at the global level suffer from vicarious trauma and they are likely to encounter voyeuristic distress ( $\mathrm{Li}$ et al., 2020). Most studies report a high prevalence of anxiety (30-70\%) and depressive symptoms (20-40\%) (Xue-Hui F et al., 2020; Chew N et al., 2020). Other reported issues included insomnia, burnout, emotional exhaustion and somatic symptoms.

\subsection{COVID-19 Exposure}

Healthcare professionals working in the first line of care, with higher clinical responsibilities and those who have been infected, have had higher prevalence of depression and anxiety symptoms (Xue-Hui F et al., 2020). The major cause of distress was noted to be, the fear of infecting their colleagues and families (Dai Y et al., 2020). The HP's have gone through tough situations during the pandemic, some HPs were quarantined or some needed hospitalization, and had to make difficult decisions, some experienced vicarious trauma for e.g. dying patients, ill patients, distressed families of the patients, etc. Major impact being lack of familial support as a preventive measure for their wellbeing. The degree of contact was directly related and the access to psychological health was inversely related to the proportion of mental health disturbances (Kang et al., 2020).

Epidemiologically, the likelihood of developing symptoms is related to the impact of the pandemic in each territory and it's staging in that territory. Increase in number of cases and reported deaths were positively correlated with declination of mental health status of the HP's in that area. Increase in number of cases is directly proportional with higher prevalence of anxiety and depression (Zhang SX et al., 2020; Naser AY et al., 2020; Dai Y et al., 2020).

A study from India showed that positive motivational factors like supportive and proud family and colleagues, positive role models, appreciation by peers and patients, a sense of validation of existence, knowledge and acceptance of the possible inevitability of infection need to be strengthened to boost the morale of the healthcare professionals. The negatives associated with patient care include stigma, multiple needs of the patients, lack of knowledge and resources, complexity of the disease and need for clear management plans. Multidisciplinary teams and screening questionnaires were the most popular opted solution for this (Mohindra et al., 2020).

\subsection{Socio-demographic Variables}

Age group and gender of the HPs influenced the subject matter of worry. Various studies support that mean age of the medical staff, ranged between $26-40$ years and females were a predominant population amongst the medical staff $(68.6 \%$ $85.5 \%$ ). A relationship between the age and depressive symptoms was studied: younger staff ( $<30$ years) had higher self-rated depression scores than the older age ( $>30$ years) (Liang et al., 2020).

Health professionals aged 31-40 years were significantly worried about infecting their families whereas those aged $>50$ were stressed due to patient's death and staff aged 41-50, other factors regarding their own safety was a matter of concern (Cai et al., 2020). Younger HPs were more afraid of contagion while older HPs were also stressed about the risk of death (Bettinsoli ML et al., 2020). Also, the older staff reported increase in stress due to fatigue and exhaustion caused by prolonged working hours and lack of personal protective equipment. The middle-aged HPs were somewhat protected against distress. In addition to the above-mentioned factors, lack of treatment and little knowledge of COVID-19 were perceived as stress inducing factors (Cai et al., 2020).

Frontline health workers in Wuhan (China) reported severe anxiety, depression and insomnia (Lai et al., 2020). Nurses compared to doctors had more anxiety and depressive symptoms. Nurses felt more anxious, compared to other professionals. Continuous fear of being infected, either themselves or their relatives was seen amongst the childbearing staff. Social support was consistently regarded as a protective factor, reducing the risk of experiencing mental stress during the pandemic.

Personality traits, such as neuroticism, previous mental disorders or physical complaints, feeling of despair or loneliness, have been found to increase the likelihood of altered mental health. Extraversion, self-efficacy or parental attachment style, were found to foster resilience (Xiao $\mathrm{H}$ et al., 2020; Wu W et al., 2020). A little is known yet about selftreatment with legal drugs (e.g. sedatives, opiates), alcohol or illegal drug use by HPs during the pandemic as a maladaptive coping strategy and the risk of developing disorders of substance use.

\subsection{Psychological Variables}

Social support given to the medical staff plays an important role and studies suggested its strong association with efficacy, sleep quality, degrees of anxiety and stress. It resulted in low levels of stress and anxiety, and increased their self-efficacy; however, no relation was found between social support and sleep quality (Xiao et al., 2020). Social and moral responsibility, recognition from hospital authorities and anticipated additional financial compensation are few of the reasons for continuing work during the outbreak. The most important stress triggering factors in the medical staff were concerns for personal safety, concern for their families and patient mortality (Cai et al., 2020).

A study conducted in India revealed that, HPs reported certain personal fears and worries regarding several factors, which included possibility of being source of infection, being quarantined (isolated), putting other staff and family at risk, fear of household problems, fear of improper use of personal protective equipment and issues due to medical insurance and lockdown. Increase of manpower, counselling, social support and better community awareness to reduce stigma were the possible solutions proposed to deal with these issues (Mohindra et al., 2020).

\subsection{Public Health Policies And Human Resources}

During the pandemic, a wider analysis should include information on the extent to which each government achieved data transparency and designed a clear and effective prevention and treatment plan. The material resources, like shortage of personal protection equipment has been associated with fear of contagion among HPs, especially to those at the first line of care (Xue-Hui F et al., 2020; Mo Y et al., 2020). On the other hand it was observed the providing resting places and adequate time to sleep, either at work locations or adapted hostels to the healthcare workers, contributed to lessen the impact of physical and emotional exhaustion and offered effective psychological support during the outburst of the pandemic (Cai H et al., 2020). 
Increased professional responsibility and immediate, close contact with infected patients, increased the likelihood of suffering from mental distress (Bettinsoli ML et al., 2020). Higher exposure rates to COVID-19 were observed amongst those in the first line of care: primary care personnel, emergency departments, intensive care units, COVID-19 ward units and support ambulance staff. Excessive hours on duty increased the risk of developing insomnia and stress (Lui X et al., 2020; Xiao $\mathrm{H}$ et al., 2020). However, amid all the hustle, it would be very interesting to know, the impact of having to rapidly acquire expertise to attend COVID-19 patients, psychological support resources, converted medical professionals, reinforcement staff and new team formations.

\subsection{Coping And Psychological Care Needs}

Measures taken by the medical staff to come on terms with or the coping measures: strict protective measures, knowledge of virus transmission and prevention against it, social isolation measures, social support and positive self-attitude (Cai et al., 2020). Medical and nursing staff with mild or subthreshold disturbances preferred to obtain services from media resources, while staff with heavier burdens wanted to seek help from the professionals directly (urgent desire to seek help from psychotherapists and psychiatrists) (Kang et al., 2020).

\section{DISCUSSION}

Epidemics and pandemics are a periodic phenomenon and have an intense impact, which may adversely affect the mental well-being of a given population. Such situations present to us several challenges. Lack of awareness often leads to unconcerned attitude, which adversely affect the preparedness to meet these challenges. Also, the fear and anxiety related to the pandemic may influence the behavior of people in the community. The current review particularly suggests that healthcare workers are encountering a considerable degree of stress, insomnia, anxiety, depression due to the COVID-19 pandemic.

COVID-19 outbreak has confronted many HPs with unexpected, life threatening experiences for which they had not been trained. Although the HPs are used to witnessing trauma and regularly deal with loss, but the high morbidity and mortality rates of the pandemic and the absence of effective treatment, have changed their normal scenario (Gerada C et al., 2020). Features specific, responsible for the mental health problems include the speculations about its mode of transmission, rapidity of spread and lack of definitive treatment protocols or vaccine on the immediate horizon, shortage of personal protective equipment, fear of family members becoming infected and new restrictive public health policies that were implemented with immediate effect. Compared to the SARS outbreak, widespread global connectivity and extensive media coverage lead to catastrophic reactions secondary to the outbreak (Tang et al., 2020; Ho et al., 2020).

In general population, the epidemic can cause severe and variable psychological effects, it may lead to the development of new psychiatric symptoms or worsening of pre-existing illnesses. People harbor a fear of falling ill or dying, excessive worry, anxiety and helplessness. Therefore, during the pandemic, most of the population including the HPs experienced unpleasant emotions, including fear, hyperarousal, intrusive memories and insomnia as well as some related to sadness or emotional exhaustion. It was seen that uncertainty was directly proportion to mental distress. Also, those quarantined tend to feel more anxious, frustrated and isolated (Gomez-Duran EL et al., 2020). Psychiatric illnesses developed include depression, anxiety, panic attacks, somatic symptoms and posttraumatic stress disorder, symptoms to delirium, psychosis and even suicidality (Sim et al., 2020; Muller, 2014; Hall et al., 2008).
With respect to the long-lasting mental health consequences of the pandemic, alcohol and substance misuse were reported by HP's months and years after the SARS outbreak, mainly among the high-risk exposure and those who needed to be quarantined, although lower among those with altruistic acceptance of risk during the outbreak or with higher social support (Wu P et al., 2020). In fact, a big concern is that the HPs may be reluctant to ask for help if needed. Initial defense mechanisms, self-treatment, rationalization, denial or minimization, used to confront stressful situations, may result in not seeking appropriate help when developing a mental disorder. The tendency might have changed during the ongoing COVID-19 pandemic. HPs received social recognition together with the mass and social media diffusion of their testimonies, lowered the internal psychological barriers to seeking professional aid if necessary (Lu Wet al., 2020). The effect of social support and coping was shown by a systematic review conducted on the impact of a disaster on the mental health of HCW. As per the report, lack of social support, communication, lack of training and maladaptive coping were the common risk factors for developing mental and psychic morbidities (Naushad et al., 2019).

Focus of the authorities continues to remain in biological and physical domains of the population, neglecting the unmet psychological needs. The Chinese government took certain initiatives to handle the trauma, by issuing emergency psychological crisis intervention guidelines for the population infected with COVID-19 (National Health Commission of the People's republic of China, 2020). Other strategies implemented included use of shift duties, online platforms with medical advice and setting up psychological intervention teams. Each psychological intervention team included: the psychosocial response team, psychological intervention technical support team, psychological intervention medical team and psychological assistance hotline teams (Kang et al. 2020).

\section{CONCLUSION}

Health authorities should consider setting up multidisciplina ry mental health response teams at regional and national levels to provide mental and psychological support to both patients and HCW. Steps to support mental health of medical staff.

a) Set up a Special Purpose Vehicle: It should be led by an executive administrator and should be multidisciplinary and include physicians, nurses, psychiatrists, psychotherapists, social workers, etc. The mission is to identify the needs of medical staff and provide the necessary support to reduce stress.

b) Awareness and communication policy: Setting up a clear line of communication is important to prevent wide-spread panic and increase compliance with pandemic response policies among both the public and medical staff. Training courses and lectures should be organized to provide up-to-date knowledge and information about threats offered by COVID-19 and its prevention. The guidance should be made fathomable, visually attractive and easily accessible

c) Monitoring of mental and physical health status (screening): mental and physical health should be equally taken into consideration. Online portal and screening algorithm must be established, for the purpose of self-reporting individual's status on duty and at home. Individuals with higher exposure to risk should be identified and screened more frequently. Relaxation technique training can be provided to help manage the stress and to improve sleep quality (Walton M et al., 2020; Spoorthy M.S. et al., 2020).

d) Provide help: formal and informal psychological support to help deal with stress. Hotline should be established that allows emergency access to psychosocial assessment and counselling services. Staff members should be 
provided with 24-hour free access to food and beverages in order to reduce risks of contamination to and from individuals. Support staff quarantined at home, remotely to alleviate loneliness, must keep in touch with the team. Emphasis on encouraging the individuals to stay in contact with friends and family (Walton M et al., 2020; Shanafelt T et al., 2020; Spoorthy M.S. et al., 2020).

e) Support from Hospital Administration: Enough stockpile of PPE should be ensured and must be distributed responsibly and reasonably. The Hospital Administrators should personally visit the frontline staff, show gratitude and honor their hard work (Walton M et al., 2020).

Timely addressal of mental health issues by psychotherapeu tic means based on stress adaptation model is important (Folkman and Greer, 2020; Xiang et al., 2020).

The psychobiological underpinnings of acute and chronic responses to trauma are diverse. Nevertheless, a comprehe nsive approach to trauma should include the specific psychosocial context within which the response to trauma is embedded. Human response to trauma is collectively modulated by coverage of basic needs, socio-cultural narratives, communitarian beliefs such as ideologies or religions and social support. Throughout the history, mankind has suffered many traumatic experiences, resilience is not an exception but fortunately, it is the most common response to suffering. The paradox: the only time most people feel alive is when they're suffering, when something overwhelms or destroys their ordinary, careful armor, the inner being is flung out onto the world.

\section{FUNDING}

The authors received no financial support for the research, authorship, and/or publication of this article.

Conflict of interest: The authors declare that there is no conflict of interest.

\section{REFERENCES}

a) Cai, H., Tu, B., Ma, J., Chen, L., Fu, L., Jiang, Y., Zhuang, Q., 2020. Psychological impact and coping strategies of frontline medical staff in Hunan between January and March 2020 during the outbreak of coronavirus disease 2019 (COVID19) in Hubei, China. Med. Sci. Monit. 26, e924171.

b) Folkman, S., Greer, S., 2000. Promoting psychological well-being in the face of serious illness: when theory, research and practice inform each other. Psychooncology 9, 11-19.

c) Kang, L., Li, Y., Hu, S., Chen, M., Yang, C., Yang, B.X., et al., 2020. The mental health of medical workers in Wuhan, China dealing with the 2019 novel coronavirus. Lancet Psychiatry 7, el4.

d) Liang, Y., Chen, M., Zheng, X., Liu, J., 2020. Screening for Chinese medical staff mental health by SDS and SAS during the outbreak of COVID-19. J. Psychosom. Res. 133, 1101-1102.

e) Mohindra, R., R.R, Suri, V., Bhalla, A., Singh, S.M., 2020. Issues relevant to mental health promotion in frontline health care providers managing quarantined/isolated COVID19 patients. Asian J. Psychiatry 51, 102084

f) Müller, N., 2014. Infectious diseases and mental health. In: Sartorius, N., Holt, R.I.G., Maj, M. (Eds.), Key Issues in Mental Health. S. KARGER AG, Basel, pp. 99-113. Available at: https://www.karger.com/Article/FullText/365542.

g) National Health Commission of the People's republic of China. The guidelines of psychological crisis intervention for COVID-19 pneumonia. Available at: http://www.nhc.gov.cn/jkj/s3577/202001/ 6adc08b966594253b2 b791be5c3b9467.

h) Naushad, V.A., Bierens, J.J., Nishan, K.P., Firjeeth, C.P., Mohammad, O.H., Maliyakkal, A.M., et al., 2019. A systematic review of the impact of disaster on the mental health of medical responders. Prehospital Disaster Med. 34, 632-643.

i) World Health Organization, 2020. Statement on the Second Meeting of the International Health Regulations (2005) Emergency Committee Regarding the Outbreak of Novel Coronavirus (2019-nCoV). Published January 30.

j) Wu, K.K., Chan, S.K., Ma, T.M., 2005a. Posttraumatic stress after SARS. Emerg. Infect. Dis. 11, 1297-1300.

k) Wu, K.K., Chan, S.K., Ma, T.M., 2005b. Posttraumatic stress, anxiety, and depression in survivors of severe acute respiratory syndrome (SARS). J. Trauma. Stress 18, 39-42.

1) Xiang, Y.T., Yang, Y., Li, W., Zhang, L., Zhang, Q., Cheung, T., et al., 2020. Timely mental health care for the 2019 novel coronavirus outbreak is urgently needed. Lancet Psychiatry 7, 228-229.

m) Xiao, H., Zhang, Y., Kong, D., Li, S., Yang, N., 2020. The effects of social support on sleep quality of medical staff treating patients with coronavirus disease 2019 (COVID-19) in January and February 2020 in China. Med. Sci. Monit. 26, e923549.

n) Zhu, N., Zhang, D., Wang, W., Li, X., Yang, B., et al., 2020. A novel coronavirus from patients with pneumonia in China, 2019. New Engl. J. Med. 382, 727-733.

o) World Health Organization (WHO) Coronavirus Disease (COVID-19) Dashboard. https://covid19.who.int/ (24May 2020, date last accessed). p) Go'mez-Duran EL, Martin-Fumado' C, Garcl'a Forero C. The psychological impact of quarantine on healthcare workers. Occup Environ Med 2020. doi:10.1136/oemed-2020-106587.

q) Bettinsoli ML, Di Riso D, Napier JL, Moretti L, Bettinsoli PF, Deimedico M, et al. Psychological impact and contextual factors associated with physical and mental health conditions of Italian Healthcare Professionals during the COVID-19 disease outbreak. PsyArXiv 2020. doi:10.31234/osf.io/w89fz.

r) Xue-Hui F, Wu L, Lun-Shan L, Kan X-H, Wang H, Yang-jun X. Analysis of mental health status and needs of health care workers in designated medical institutions of tuberculosis during the epidemic period of COVID-19. Research Square [Preprint] 2020; doi:10.20203/rs-22402/vl.

s) Lu W, Wang H, Lin Y, Li L. Psychological status of medical workforce during the COVID-19 pandemic: a cross-sectional study. Psychiatry Res 2020; 288:112936.

t) Liu X, Liang S, Zhang R, Wei Y, Li J, Wang C, et al. Perceived social support and its impact on psychological status and quality of life of medical staffs after outbreak of SARS-CoV-2 pneumonia: a cross-sectional study. Lancet 2020; doi: 10.2139/ssm.3541127.

u) Xiao H, Zhang Y, Kong D, Li S, Yang N. The effects of social support on sleep quality of medical staff treating patients with coronavirus disease 2019 (COVID-19) in January and February 2020 in China. Med Sci Monit 2020; 26:e923549.

v) Dai Y, Hu G, Xiong H, Qiu H, Yuan X. Psychological impact of the coronavirus disease 2019 (COVID-19) outbreak on healthcare workers in China. Lance Psychiatry 2020; doi: 10.1 101/2020.03.03.20030874

w) Cai H, Tu B, Ma J, Chen L, Fu L, Jiang Y, et al. Psychological impact and coping strategies of frontline medical staff in Wuhan between January and March 2020 during the outbreak of coronavirus disease 2019 (COVID-19) in Hubei, China. Med Sci Monit 2020; 26:e924171.

x) Wu W, Zhang Y, Wang P, Zhang L, Wang G, Lei G, et al. Psychological stress of medical staffs during outbreak of COVID-19 and adjustment strategy. J Med Virol 2020; doi: 10.1002/jmv.25914.

y) Mo Y, Deng L, Zhang L, Lang Q, Liao C, Wang N, et al. Work stress among Chinese nurses to support Wuhan in fighting against COVID-19 epidemic. J Nurs Manag 2020; doi: 10.1111/jonm.13014.

z) Cao J, Wei J, Zhu H, Duan Y, Geng W, Hong X, et al. A study of basic needs and psychological wellbeing of medical workers in the fever clinic of a tertiary general hospital in Beijing during the COVID-19 outbreak. Psychother Psychosom 2020; 30:1-3.

aa) Naser AY, Dahmash EZ, Al-Rousan R, Alwafi H, Alrawashed HM, Ghoul I, et al. Mental health status of the general population, healthcare professionals, and university students during 2019 coronavirus disease outbreak in Jordan a crosssectional study. MedRrviv [Preprint] 2020; doi: 10.1101/2020.04 09 . 20056374.

bb) Zhang SX, Liu J, Jahanshahi A, Nawaser K, Yousefi A, Li J, et al. At the height of the storm: Healthcare staff's health conditions and job satisfaction and their associated predictors during the epidemic peak of COVID-19. Brain Behav Immun 2020. doi:10.1016/j.bbi.2020.05.010.

cc) Chew N, Lee G, Tan B, Goh Y, Ngiam N, Yeo L, et al. A multinational, multice ntre study on the psychological outcomes and associated physical symptoms amongst healthcare workers during COVID-19 outbreak. Brain Behav Immun 2020. doi:10.1016/j.bbi.2020.04.049.

dd) Gerada C. Doctors and their defences. BMJ 2019; 364:1871

ee) Wu P, Liu X, Fang Y, Fan B, Fuller CJ, Guan Z, et al. Alcohol abuse/dependence symptoms among hospital employees exposed to a SARS outbreak. Alcohol Alcohol 2008; 43:706-12.

ff) Wu P, Fang Y, Guan Z, Fan B, Kong J, Zhongling Y, et al. The psychological impact of the SARS epidemic on hospital employees in China: exposure, risk perception, and altruistic acceptance of risk. Can J Psychiatry 2009; 54:302-11.

gg) Walton M., Murray E., Christian M.D. Mental health care for medical staff and affiliated healthcare workers during the COVID-19 pandemic. Eur Heart I Acute Cardiovasc Care. 2020;9(3):241-47. [PMC free article][PubMed][Go ogle Scholar]

hh) Shanafelt T., Ripp J., Trockel M. Understanding and addressing sources of anxiety among healthcare professionals during the COVID-19 pandemic. J Am Med Assoc.2020;323(21):2133-2134.[PubMed] [Google Scholar]

ii) Spoorthy M.S., Pratapa S.K., Mahant S. Mental health problems faced by healthcare workers due to the COVID-19 pandemic-a review. Asian J Psychiatr. 2020:51:1-4. [PMC free article][PubMed][Google Scholar]

jj) Coronavirus Pandemic. 2020. Coronavirus Pandemic. https://www.worldomet ers.info/coronavirus/ (Accessed on April 27, 2020) [Google Scholar]

kk) Ho C.S., Chee C.Y., Ho R.C. Mental health strategies to combat the psychol ogical impact of COVID-19 beyond paranoia and panic. Ann. Acad. Med. Singapore. 2020;49(1):1-3. [Abstract] [Google Scholar]

11) Li Z., Ge J., Yang M., Feng J., Qiao M., Jiang R....Zhou Q. Vicarious traumatization in the general public, member and non-members of medical teams aiding in COVID-19 control. Brain behavior, and immunity Behav. Immun2020 [Europe PMC free article] [Abstract] [Google Scholar].

$\mathrm{mm}$ ) WHO; 2020d WHO Coronavirus Disease 2019 (COVID-19) Situation Report70 (2020) Google Scholar. 